\title{
Post Cardiac surgery Home-Monitoring System
}

\author{
Efthyvoulos Kyriacou ${ }^{1,2}$, Panayiota Chimonidou ${ }^{1}$, Constantinos Pattichis ${ }^{1}$, \\ Ekaterini Lambrinou $^{3}$, Vassilis I. Barberis ${ }^{4}$, George P. Georghiou ${ }^{4,5}$ \\ ${ }^{1}$ University of Cyprus, ${ }^{2}$ Frederick University Cyprus, \\ ${ }^{3}$ Cyprus University of Technology, \\ ${ }^{4}$ American Heart Institute Cyprus, ${ }^{5}$ Tel Aviv University Israel \\ \{e.kyriacou@frederick.ac.cy, pattichi@cs.ucy.ac.cy\}
}

\begin{abstract}
One of the major factors limiting but also causing the application of modern technology in medicine is the response time to patients, should they need specialized medical care. In this work we propose a solution that is aiming to help and improve the patients' life after a cardiac surgery. This is a group of patients that have to be monitored for a short period after the operation. The aim of this project is to give the opportunity to these patients to be monitored at home after their operation so as to keep their rehabilitation period as smooth as possible and avoid unnecessary visits to the hospital or prevent adverse events. This is going to be achieved by creating an integrated web- based system which can regularly monitor several biosignals like ECG, blood pressure, arterial $\mathrm{SpO} 2$ and parameters like body weight and provide the tools to identify any unusual events.
\end{abstract}

Keywords: Cardiac surgery patients, home monitoring, biosignals monitoring, web based telemedicine systems.

\section{Introduction}

Cardiovascular disease (CVD) is the leading cause of death and adult disability in the industrial world. Despite advances in the diagnosis and treatment of coronary heart disease (CHD), it remains the single most frequent cause of death in Americans older than 65 years old [1]. International statistics parallel those of the USA with an increasing epidemiology of CHD occurring worldwide [2]. A recent study by the World Health Organization revealed that by 2015 almost 20 million people will die from CVD, mainly from CHD and stroke [3]. Coronary artery bypass graft (CABG) surgery is the most frequently performed major surgery for CHD in the United States [2].

As the prevalence of CVD is increasing, new strategies need to be developed in order to reduce the cost of health care services and at the same time to improve patients' quality of life. As a result, there is an increasing interest in care delivery models that incorporate information-communication technology, with the transfer of physiological data [such as blood pressure, weight, electrocardiogram (ECG), 


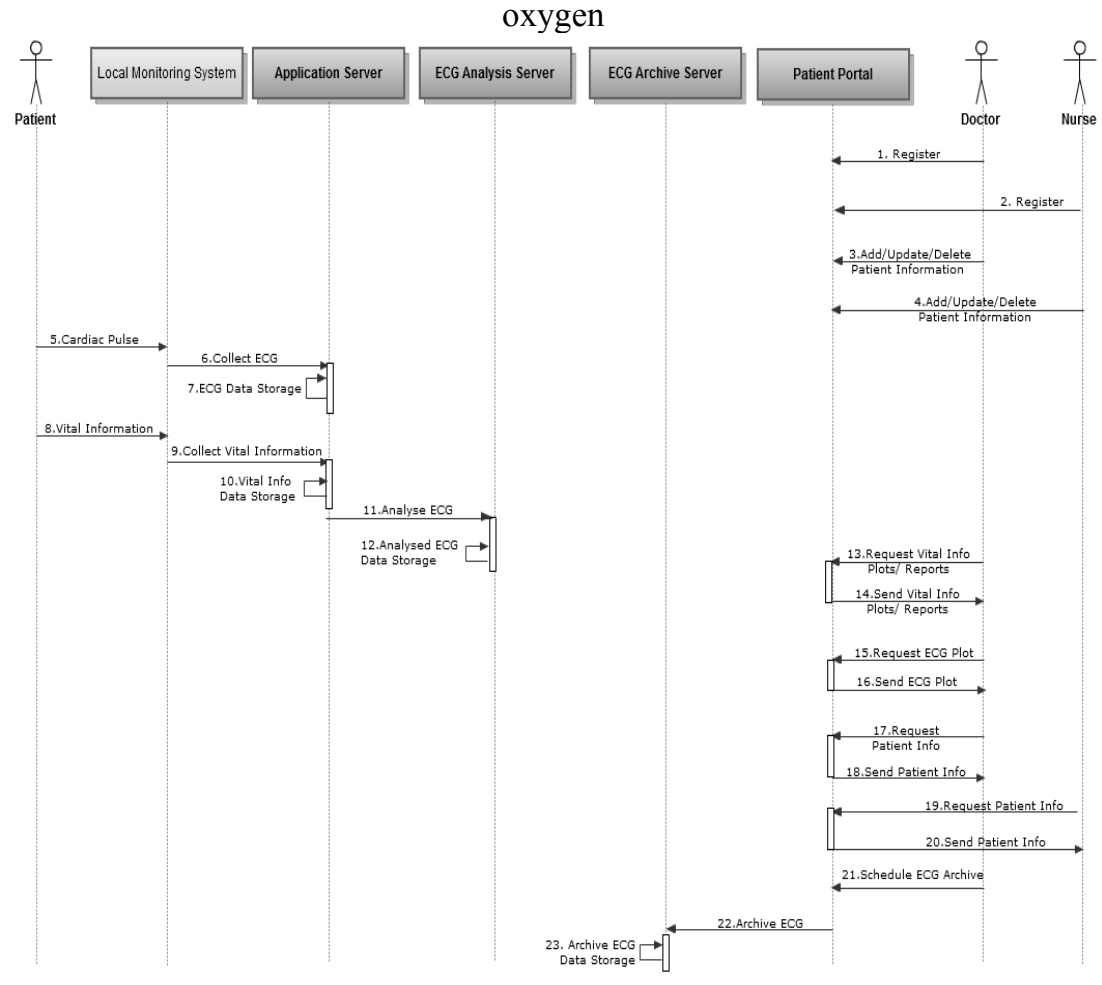

Fig. 1: Portal Sequence Diagram

saturation], via the internet, the mobile phones, the wireless communications from home to health care providers.

The production of revolutionary medical monitoring systems we are proposing includes a system that will enable physicians to monitor patients undergoing cardiac surgery after hospital discharge.

A considerable proportion of patients experience adverse events or are readmitted within a few weeks after discharge, leading to worse outcomes and/or increased costs. These problems are likely to increase over the next few decades as the longevity of the population increases [4- 6]. The impact of structured telephone support on the risk of readmissions and rehospitalization after cardiac surgery have been proved in several studies. These systems proved to be useful from the actual monitoring point of view as well as the psychological point of view. They also give the opportunity for significant savings in the health care system, improvements to patient's care and at the same time, to patients' health related quality of life [7-11].

Like every web-based application, in order to achieve a respectable level of status, the system must offer substantial services. Some typical facilities that our system includes are: doctor registration and collaboration, patient's ECG monitoring and automatic analysis in order to extract useful information for the physicians. This method of management of patients after discharge for cardiac surgery can be very 
beneficial in combating episodes of exacerbation and preventing rehospitalizations, urgent care visits, and emergency room visits.

The other important objective of this system is to help patients. With the use of this system a patient will not have to visit the hospital almost every day just for some minutes or whenever thinking that something is wrong. The impact of the system support on the risk of readmissions can be attributed in part to the triage of patients at the first sign of clinical deterioration by the specialist nurse, and the consequent immediate intervention of a primary care physician. Alternatively with the help of specialized sensors and other equipment, involving daily transmission of vital signs (eg ECG, blood pressure, and oxygen saturation), symptoms and weight lead to earlier detection and management of clinical deterioration by both the patient and/or the health care professional.

The current project was build upon and further past research [12], incorporating multiple, disparate technologies in one cohesive system, focusing on the reasoning and inferencing components of the system via the application of innovative machine learning techniques, transferring this into a viable home based product that can handle this complex domain and meet the needs of patients at need.

\section{Methodology}

\subsection{System's Purpose and System Functions}

The system will gather all the necessary information concerning patients, such as weight, blood pressure, oxygen saturation and ECG. The ECG signal is automatically analyzed by the system in order to identify possible arrhythmias and facilitate extra and helpful information to the doctor. Each doctor can see his patients' information; on the other hand, he must have the ability to give or remove access to other doctors, in order to enable collaboration between them and help each other to get some decisions.

The web portal provides site functionality such as historical information storage and maintenance. Medical background information, patient hospitalization information, and patients' symptoms data. Questionnaires completed before and after the treatment in order to calculate Physical, Emotional, Global and Sum Score, finally patient's calls and follow up information.

Furthermore the system is used to store, analyze and archive patients' information. The system is able to facilitate and provide all the necessary functionality to the doctors and the nurses. An overall sequence diagram showing the sequence of actions being done from the users (Patient, Doctors, and Nurses) can be seen in fig. 1. 


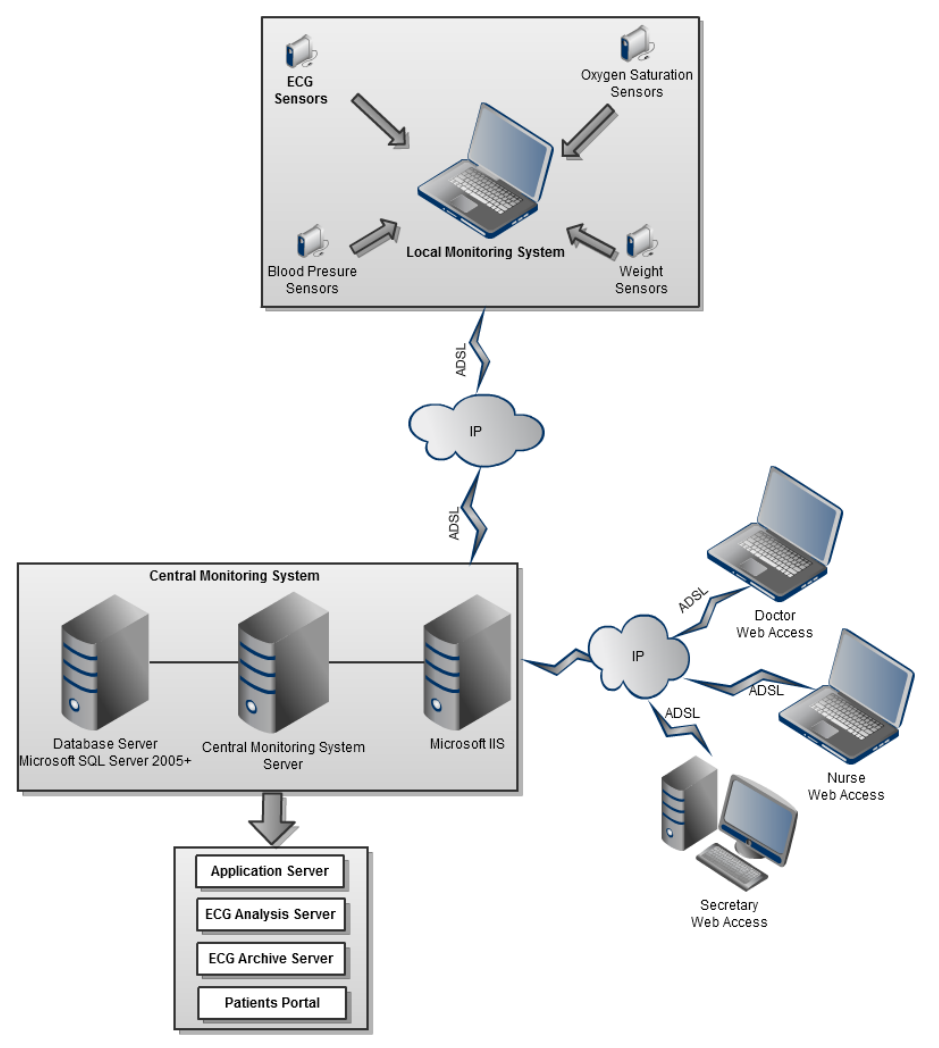

Fig.2: Overall System Architecture

\subsection{Basic System operations and functions}

In general the System facilitates the following operations and functions:

- Doctor registration

- Patient Registration which can be done by the doctor or the nurse

- Doctors have the ability to give access to another doctor as contributor or just as a viewer.

- Before and after patient's treatment several scores are measured based on predefined questionnaires.

- Historical information relating to previous diseases, medical background and symptoms are handled.

- Patient calls and follow-up information are handled by the system.

- Several statistics about biosignals and overall graphs of other parameters can be provided to the doctor. These refer to:
o ECG
o Weight
o Blood pressure 
o Oxygen saturation

o Score statistics

- Automatic analysis of ECG signals for possible arrhythmia detection. The analysis is being done based on an open source arrhythmia detection algorithm provided by E.P Limited [14]

- The doctor has the ability to schedule an archive process for the patient's ECG.

\subsection{System Architecture}

The overall architecture of the system can be seen in fig.2. It's actually a web based application and mainly consists of the central web server and the local monitoring systems. The communication between the two systems is being done using a dedicated web service and predefined XML message over the IP network.

The Local Monitoring System consists of a dedicated PC with the appropriate software, ECG sensors, Blood pressure, Oxygen Saturation sensors and a digital weight scale. This unit is responsible for collecting and uploading all vital information from the patient. Currently we are using a Dyna-Vision(C) (DVM-010S) ECG and $\mathrm{SpO}_{2}$ acquisition device [13] (connected with the pc using Bluetooth or USB) and CONTEC08A (CONTECC Medical Systems) for Non Invasive Blood Pressure.

The Central Monitoring Web Server collects the vital information from the Local Monitoring Systems. It includes four major processes. The first one is the Application Server which is the central process that is used to collect and store vital information and side information. The second one is the ECG Analysis Server which is used to analyze (detection of possible cardiac arrhythmias) and store the ECG. The third major process is the ECG Archive Server which is used to archive the ECG that was scheduled by the doctor. Finally, the most important process is the Patients Portal, which is used to collect and display the patients' information and their vital information. The portal is accessible through a web interface. Doctors, nurses, secretaries and patients can apply their user name and password and in this way they are able to maintain and view the patients' information. Security of the system was developed using different access levels for each group. It is being done by using a Microsoft Membership provider from ASP.NETC [16]. Password code of each user is Hashed using a one-way hashing algorithm and a randomly generated "salt" value.

A snapshot of the initial screen of the system can be seen in fig. 3 where on this screen a user doctor can see all recent uploads from his patients' and select any subsection to see either past medical information, follow up, vital info etc related to any of his patients. 


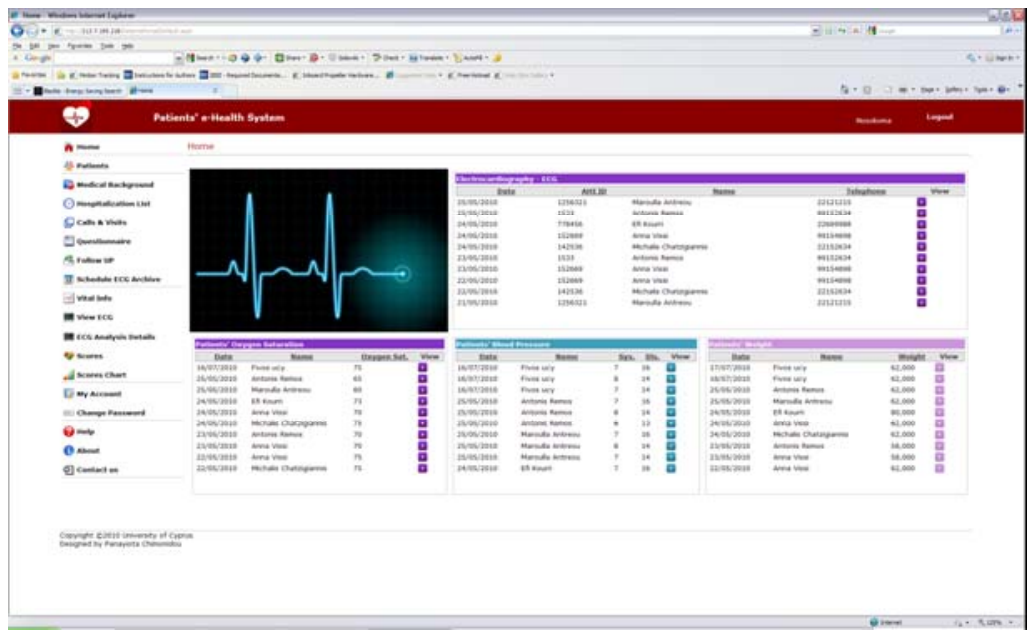

Fig. 3. Initial system screen displaying patients that have recently uploaded information and links to several screens of the system.

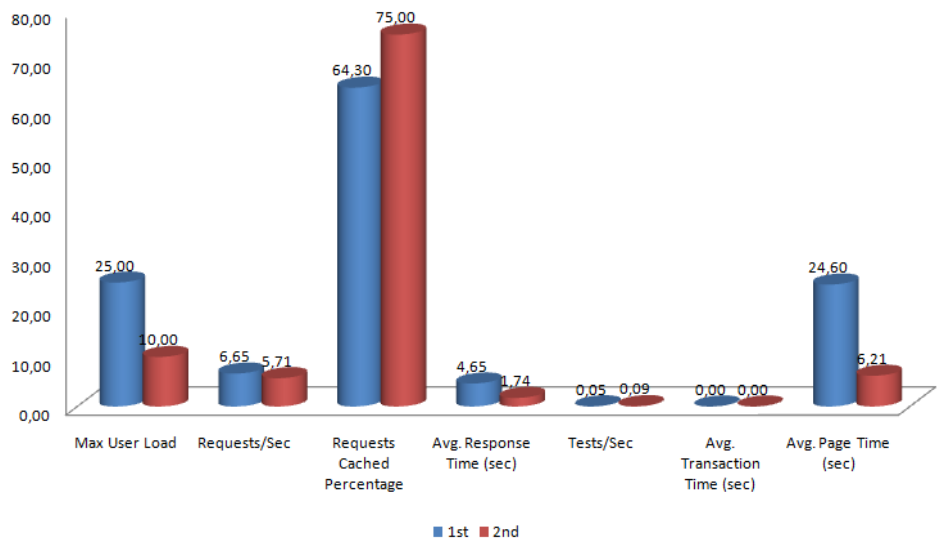

Fig. 4. Overall testing results

\subsection{Tools used for system implementation}

For the implementation of the system we had used the Microsoft Visual Studio 2008 with ASP.NET and Visual Basic.NET [15-16], as the main programming tool. Microsoft SQL Server 2008 was used as the database management system. Furthermore, Ajax Toolkit 3.5 was used for several enhancements on the user interface. Finally for the ECG graphs over the web, the National Instruments Measurements Studio was used [17]. 


\section{Results}

Professional statistics show that satisfactory project evaluation depends heavily on a thorough testing plan and strategy. Therefore, rigorous testing is demanded for the effective evaluation of the finished end-product. Successful testing of the system's performance was required at each of the following levels:

Module Testing. For this type of testing, each individual module was tested to determine whether all the system's modules are logically and functionally correct. If an error occurred, a suitable debugging procedure was followed.

Integration Testing. This form of testing was applied to all interfaces among modules to ensure that transfer of both data and control were performed correctly. This testing method included the run-time evaluation of all variables that are passed from one module to another.

Function Testing. The results and outcomes of the program were evaluated after the execution of the program in which every functional menu selection had been activated systematically in turn.

Final System Testing. The objective of this type of testing was the full test of the entire system on the completion of the system development period. In order to achieve this several testing tools e.g. the load test, the unit test that the visual studio 2008 offers helped us with testing in order to discover programming errors and potential problems. The system was tested with simulations for data input, retrieval and ECG automatic analysis. The results for 25 and 10 users running for 10 minutes gave a warm-up duration of 30 and 60 seconds respectively. Tests were applied to the pages that have the most significant processing functions like ECG Preview and VitalInfo

The graphical representation of overall results can be viewed in fig. 4. Blue bars display the first case that the scenario was simulating 25 simultaneous accesses to the Patients Portal and the red bars indicate the scenario with 10 users. As expected the results of the second case are much better than the first case. Request cached, Average Response Time and the Average Page time are better in the case that we have 10 users accessing the patient portal. The "average page time" from 24.60 seconds it is decreased to 6.21 seconds. In any case the results depend on the server lines and hardware used.

\section{Conclusions}

These are the initial steps followed in order to create a system that will be used for monitoring patients after cardiac surgery. The system has been completed and the next step will be the actual system and acceptance testing before moving into the final use of the system in real cases. The system will be tested with real data, with the patients' and doctors' help. The goal is to use this system to the minimum number of 50 patients initially, in order to ensure that everything is working correctly and it satisfies all requirements. 


\section{References}

1. American Heart Association, Heart disease and stroke statistics-2008, update, Dallas, Texas, 2007, http://www.americanheart.org/presenter.jhtml.

2. Kleinpell RM and Avitall Boaz : Integrating telehealth as a strategy for patient management after discharged for cardiac surgery. Results of a pilot study. Journal of Cardiovascular Nursing, vol.22(1), (2007).

3. World Health Organisation, Fact sheet No 317, February 2007, http://www.who.int

4. Giordano, A., Scalvini, S., Zanelli, E., Corrà, U., Longobardi, G., L., , Ricci, V., A., Baiardi, P., Glisenti, F.: Multicenter randomised trial on home-based telemanagement to prevent hospital readmission of patients with chronic heart failure. International Journal of Cardiology, vol.9(131)2, pp.192-9, (2009).

5. Schwarz, K., A., Mion, L., C., Hudock, D., Litman, G.: Telemonitoring of heart failure patients and their caregivers: a pilot randomized controlled trial. Progress in Cardiovascular Nursing, vol.23(1), pp.18-26, (2008).

6. Wakefield, B., J., Ward, M., M., Holman, J., E., Ray, A., Scherubel, M., Burns, T.,L., Kienzle, M., G., Rosenthal, G., E.: Evaluation of home telehealth following hospitalization for heart failure: a randomized trial. Telemedicine Journal and $\mathrm{E}$ Health, vol.14(8), pp.753-61, (2000).

7. Rodrvguez J, Dranca L, Gopi A and Illarramendi A: A Web Access to Data in a Mobile ECG Monitoring System. In: Stud. Health Technol Inform, vol.105, pp. 100111, (2004).

8. Ryan, A., McCann S., McKenna H.: Impact of community care in enabling older people with complex needs to remain at home. In: International Journal of Older People Nursing, vol.4(1), pp.22-32, (2009).

9. Glascock A., Kutzik D.: The Impact of Behavioral Monitoring Technology on the Provision of Health Care in the Home. In: Journal of Universal Computer Science, vol. 12(1), pp. 59-79, (2006).

10. Sixsmith A : An evaluation of an intelligent home monitoring system. In: Journal of Telemedicine and Telecare, vol.6(2), pp. 63-72, (2000).

11. Drennan, V., Levenson, R., Goodman, C., Evans C.: The workforce in health and social care services to older people: developing an education and training strategy. In: Nurse Education Today, vol.24, pp. 402 - 408, (2004).

12. Kyriacou E., Pattichis C., Hoplaros D., Kounoudes A., Milis M., Jossif A.: A System for Monitoring Children with Suspected Cardiac Arrhythmias Technical Optimizations and Evaluation. In: XII Mediterranean Conference on Medical and Biological Engineering and Computing, MEDICON 2010, Chalkidiki, Greece, (2010).

13. RS TechMedic BV , ECG monitors, http://www.dyna-vision.com/products.html.

14. Open Source Arrhythmia detection Software DOI - Patrick S. Hamilton, http://www.eplimited.com

15. MSDN website, http://msdn.microsoft.com/

16. The Official Microsoft ASP.NET Site, http://www.asp.net

17. National Instruments, http://www.ni.com 